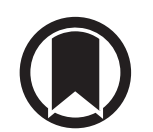

CrossMark

\title{
Current challenges in the management of nonsmall cell lung cancer brain metastases
}

\author{
Lizza E.L. Hendriks ${ }^{1}$, Jacques Cadranel $^{2}$ and Thierry Berghmans ${ }^{3}$ \\ Affiliations: ${ }^{1}$ Dept of Pulmonary Diseases, GROW - School for Oncology and Developmental Biology, \\ Maastricht University Medical Center+, Maastricht, The Netherlands. ${ }^{2}$ Service de Pneumologie, Assistance \\ Publique Hôpitaux de Paris, Hôpital Tenon and Sorbonne Université, Paris, France. ${ }^{3}$ Dept of Intensive Care \\ and Oncologic Emergencies \& Thoracic Oncology, Institut Jules Bordet, Université Libre de Bruxelles, \\ Brussels, Belgium.
}

Correspondence: Lizza E.L. Hendriks, Dept of Pulmonary Diseases, Maastricht University Medical Center+, PO Box 5800, 6202 AZ Maastricht, The Netherlands. E-mail: lizza.hendriksđamumc.nl

@ERSpublications

Brain metastases remain a major problem in NSCLC. Major improvements in systemic therapies have been observed during the past decade but their impact on brain metastases control as well as their combination with radiation techniques needs further research. http://bit.ly/2Jn4v5a

Cite this article as: Hendriks LEL, Cadranel J, Berghmans T. Current challenges in the management of nonsmall cell lung cancer brain metastases. Eur Respir J 2020; 55: 1901686 [https://doi.org/10.1183/ 13993003.01686-2019].

\section{Introduction}

In May 2019, the third European Respiratory Society research seminar of the Thoracic Oncology Assembly entitled "New biomarkers, molecules and therapeutic sequences for non-small cell lung carcinoma (NSCLC) in the era of precision medicine" was held in Paris, France. The previous two seminars of the Thoracic Oncology Assembly were on targeted therapy (2015) [1] and immune checkpoint inhibitors (ICI, 2017) [2]. During this seminar, breakout sessions on difficult situations were organised. One of the most original and useful was on the current challenges in brain metastases (BM) management, that we propose to share with European Respiratory Journal readers.

BM are associated with decreased quality of life (QoL) and poorer survival compared to patients without BM [3]. BM incidence is increasing, probably due to more screening, better imaging techniques and longer survival because of improved systemic therapies, such as tyrosine kinase inhibitors (TKIs) and ICI (with or without chemotherapy). Therefore, BM management becomes more and more important. However, BM screening and treatment practices differ, partly because of heterogeneous access to imaging and radiotherapy facilities, and heterogeneous access to newer systemic treatments [4]. Furthermore, guidelines for BM management recommendations are not consensual (reviewed by Levy et al. [4]), and high-level evidence regarding screening and treatment is scarce. Randomised trials specifically including NSCLC BM patients are largely lacking, and patients with untreated and/or unstable, or even all, BM were excluded from most clinical trials (reviewed by EL RasSY et al. [5] and МCCOACH et al. [6]).

\section{Screening}

There was consensus that BM screening for NSCLC is useful, at least for certain stages. Most session participants screened all stage III patients eligible for therapy with curative intent, and stage IV with a targetable driver alteration, while some screened stage IB and II. Magnetic resonance imaging (MRI) was 
preferred above contrast-enhanced computed tomography (CT), but lack of timely access to MRI was a problem. Brain CT during ${ }^{18} \mathrm{~F}$-fluorodeoxyglucose positron emission tomography was sometimes used, although the CT protocol was not optimised for brain imaging. Except for stage IV patients with a driver alteration, regular brain imaging follow-up was not performed if a patient did not have baseline BM. Close follow-up was usually performed if a systemic treatment was initiated in a patient with asymptomatic BM.

\section{Selection for BM treatment}

Prognostic scores (Recursive Partitioning Analysis (RPA) [7] and disease specific-Graded Prognostic Assessment (ds-GPA) [8] were mainly used by radiation oncologists. A major drawback is that these scores were validated in patients undergoing cranial irradiation (stereotactic (SRT) or whole brain (WBRT)), but these scores did not incorporate the availability of a BM-effective systemic therapy. For example, the molecular-GPA (mol-GPA) incorporates the presence of an epidermal growth factor receptor (EGFR) or anaplastic lymphoma kinase $(A L K)$ molecular alteration [9], but does not take into account whether TKI treatment options are exhausted or not. Recently, ds-GPA was associated with survival in a retrospective evaluation of 255 NSCLC BM patients treated with ICI [10]. There was consensus that performance status was a major treatment selection factor, treatment being often withheld in front of poor performance status, except in those with a targetable driver.

\section{Selection of type of treatment}

Local therapy (radiation and/or surgery) was preferred in neurologically symptomatic BM patients, although upfront TKI with good blood-brain barrier (BBB) penetration rate was discussed in patients with only mild symptoms, and without eloquent BM (i.e. growth of BM in these brain areas will result in loss of sensory processing or linguistic ability, minor paralysis, or paralysis). In asymptomatic patients, ICI and/or chemotherapy was preferred to WBRT, because of WBRT side-effects. It is known that for neurologically asymptomatic NSCLC patients treated with first line chemotherapy, WBRT can be delayed or withheld in more than half of patients (reviewed by Zimmermann et al. [11]). ICI can also be used upfront to treat selected asymptomatic BM patients, as was shown in a phase II trial. Objective response rate was similar in $\mathrm{BM}$ and extracranial disease, around $30 \%$ for programmed death ligand-1 (PD-L1) positive patients (10/34) but $0 \%(0 / 5)$ in PD-L1 negative/unevaluable patients (although some experienced a long disease control) $[12,13]$. Some participants specifically selected a first line ICI-chemotherapy combination, even in $\mathrm{PD}-\mathrm{L} 1 \geqslant 50 \%$ patients, to minimise the risk of symptomatic $\mathrm{BM}$ progression. Furthermore, the combination of ICI (pembrolizumab) and pemetrexed-platinum was superior to platinum-doublet chemotherapy in an exploratory analysis of the KEYNOTE189 trial BM subgroup [14].

TABLE 1 Summary of breakthrough session and future directions

Topic discussed in Summary management from breakout session Future directions/open questions

breakout session

Screening for BM Especially in stage III

Stage IV with driver alterations

No consensus on stage IB/II

MRI preferred

Follow-up only in BM and driver altered patients

Selection for BM treatment

Selection of treatment type
Prognostic scores mainly used by radiation oncologist

Prognostic scores do not consider available systemic treatment options

Performance status important for treatment selection

Preferably local treatment in neurologically symptomatic patients

Systemic treatment in neurologically asymptomatic patients preferred above WBRT; discussion on SRT

No consensus on treatment sequence in driver mutated patients (SRT upfront versus TKI upfront)

If available, next generation TKIs preferred in driver altered patients

No consensus on concurrent TKI and cranial irradiation
Evaluate whether screening improves QoL and OS Improved prediction models for risk of BM development needed Improved imaging techniques needed

Evaluate prognostic scores incorporating whether there are systemic treatment options available with intracranial activity

Specific trials for BM patients evaluating systemic treatment combinations le.g. ICl plus chemotherapy, and/or angiogenesis inhibition)

Evaluate best treatment strategy in randomised trials (SRT followed by systemic treatment and vice versal

Evaluate safety of concurrent TKI and cranial irradiation

BM: brain metastases; MRI: magnetic resonance imaging; QoL: quality of life; OS: overall survival; WBRT: whole brain irradiation; SRT: stereotactic radiotherapy; TKI: tyrosine kinase inhibitor; ICI: immune checkpoint inhibitor. 
Although not evaluated in a randomised trial, bevacizumab added to carboplatin-paclitaxel was mentioned as an option, based on the single arm phase II BRAIN trial $(\mathrm{N}=67)$ results, with a $61 \%$ intracranial objective response rate and a median survival of 16.0 months [15]. Furthermore, in preclinical models, bevacizumab reduces BM formation [16]. Unfortunately, BM subgroup results have not been reported for the IMPOWER 150 trial quadruplet regimen (carboplatin-paclitaxel-bevacizumab-atezolizumab) [17].

There was no consensus on the treatment sequence (SRT followed by TKI or vice versa), especially for neurologically asymptomatic EGFR-mutated patients. For EGFR, the discussion was mainly based on the retrospective series described by MAGNUSON et al. [18], in which EGFR-mutated patients with BM had a longer survival if treated upfront with SRT or WBRT, followed by EGFR-TKI, compared with upfront EGFR-TKI followed by cranial irradiation upon brain progression. For driver mutated patients there was a preference for the upfront use of next generation TKIs, such as osimertinib (EGFR) and alectinib/ brigatinib $(A L K)$, as these drugs have a superior BBB penetration rate compared with first generation TKI.

Some participants used a TKI or ICI concurrent with cranial irradiation, to minimise the risk of a disease flare (TKI) or to promote an abscopal effect (ICI). For those that did not use concurrent radiation-systemic treatment strategies, the risk of increased neurotoxicity was the main concern.

\section{Future directions}

BM management practices discussed in this breakout session generally reflected the results reported in a recent European survey [4]. Table 1 summarises the conclusions from the breakout sessions and open questions/areas for future research.

\section{BM screening: patients and techniques}

For imaging, the main differences were that in the survey only two-thirds of stage III NSCLC and $50 \%$ of driver altered patients were screened for BM, and only half of responders used MRI as the screening method [4]. However, in stage III NSCLC (with a risk of approximately $20 \%$ asymptomatic baseline BM [19]), MRI detects BM in $4.7 \%$ of neurologically asymptomatic patients, after a negative contrast-enhanced brain CT [20]. Open questions are whether BM screening improves QoL and/or survival and which stages should be screened. Besides a higher disease stage, adenocarcinoma histology, younger age and probably $A L K$-rearrangements are associated with a higher risk of BM [21-23], but these factors are not sensitive enough to select patients for baseline and/or follow-up BM screening. Up till now, blood or tissue-based biomarkers (reviewed by Pedrosa et al. [24]) are not well enough studied, and more research is needed. Another option could be to improve current imaging techniques.

\section{Optimising systemic treatment for BM}

As discussed during the breakthrough session, the combinations of different systemic treatments are interesting to specifically evaluate in NSCLC BM patients, and specific trials for NSCLC patients with BM are ongoing. Examples are ICI and chemotherapy (e.g. NCT03526900 (ATEZO-BRAIN: atezolizumabcarboplatin-pemetrexed) or chemotherapy and bevacizumab (e.g. NCT01951482: pemetrexed-cisplatin with/without bevacizumab), or TKI and bevacizumab (e.g. NCT02521051: alectinib plus bevacizumab, and NCT02971501: osimertinib with/without bevacizumab).

\section{Best sequence of local and systemic therapy}

It is unclear whether upfront local therapy (especially SRT) followed by systemic treatment, or systemic treatment followed by local therapy upon brain progression is the best treatment strategy for patients with a driver alteration. The next generation TKIs have a good BBB penetration rate, with long BM control reported in randomised phase III trials (reviewed by REMON et al. [22]). To answer this question, clinical trials such as NCT03769103 (osimertinib versus SRT followed by osimertinib) and NCT02714010 (EGFR-TKI or EGFR-TKI concurrent with WBRT) are ongoing. Interestingly, ICI can result in dissociated brain and extracranial responses [12]. A possible explanation for brain progression with extracranial response is that the brain seems to have a different immune environment. Compared with the extracranial disease sites BM more often have an immune ignorant phenotype [25] and a contraction in the number of T-cell clones despite a higher tumour mutational burden [26]. With these data, the finding that radiation can cause an abscopal effect [27], and the suggestion in retrospective series that ICI concurrent with SRT can improve survival [28], the added value of cranial radiotherapy to ICI is being evaluated. Several ICI-radiotherapy trials are ongoing (e.g. NCT02858869, NCT02696993, NCT02978404, NCT02696993, NCT03955198).

There is a lack of good quality data on the safety of combining ICI or TKI with cranial irradiation. For the EGFR-TKI erlotinib, gefitinib and icotinib phase II or III data have been reported, and the combination with WBRT seems safe, but not when also combined with SRT (reviewed by HendRIKs et al. [29]). A 
drawback is that in the majority of these trials there was no neurocognitive assessment, and there was no selection based on EGFR-mutation [29]. Retrospective series suggest that PD-(L)1 inhibitors can be safely combined with cranial irradiation, but detailed neurotoxicity assessment was lacking [30, 31]. To evaluate the question whether TKI can be combined with cranial irradiation, a prospective observational Swiss registry (TOaSTT: any type of systemic treatment) and a Dutch multicentre platform trial (NL6518/NTR6707, TKI treatment) are currently enrolling patients; for ICI, several trials are ongoing (summarised above).

\section{Conclusions}

In conclusion, BM remain a major problem in NSCLC either at diagnosis or during the disease course. Major improvements in systemic therapies have been observed during the past decade (TKI, ICI) but their impact on BM control as well as their combination with radiation techniques (WBRT, SRT) needs further research.

Conflict of interest: L.E.L. Hendriks reports other funding from Boehringer Ingelheim, BMS, Roche, Lilly and BMS; grants from Roche, Boehringer Ingelheim; grants and other funding from AstraZeneca; personal fees from Quadia, outside the submitted work. J. Cadranel reports grants from AstraZeneca, Boehringer Ingelheim, Novartis and Pfizer; personal fees from AstraZeneca, Boehringer Ingelheim, BMS, Lilly, MSD, Novartis, Pfizer, Roche and Takeda, outside the submitted work. T. Berghmans has nothing to disclose.

\section{References}

$1 \quad$ Meert AP, Cadranel J. Targeted therapy for lung cancer. Eur Respir J 2015; 46: 1239-1241.

2 Costantini A, Grynovska M, Lucibello F, et al. Immunotherapy: a new standard of care in thoracic malignancies? A summary of the European Respiratory Society research seminar of the Thoracic Oncology Assembly. Eur Respir J 2018; 51: 1702072.

3 Peters S, Bexelius C, Munk V, et al. The impact of brain metastasis on quality of life, resource utilization and survival in patients with non-small-cell lung cancer. Cancer Treat Rev 2016; 45: 139-162.

4 Levy A, Faivre-Finn C, Hasan B, et al. Diversity of brain metastases screening and management in non-small cell lung cancer in Europe: results of the European Organisation for Research and Treatment of Cancer Lung Cancer Group survey. Eur J Cancer 2018; 93: 37-46.

5 Rassy EE, Botticella A, Kattan J, et al. Non-small cell lung cancer brain metastases and the immune system: From brain metastases development to treatment. Cancer Treat Rev 2018; 68: 69-79.

6 McCoach CE, Berge EM, Lu X, et al. A brief report of the status of central nervous system metastasis enrollment criteria for advanced non-small cell lung cancer clinical trials: a review of the clinicaltrials.gov trial registry. J Thorac Oncol 2016; 11: 407-413.

7 Gaspar L, Scott C, Rotman M, et al. Recursive partitioning analysis (RPA) of prognostic factors in three Radiation Therapy Oncology Group (RTOG) brain metastases trials. Int J Radiat Oncol Biol Phys 1997; 37: 745-751.

8 Sperduto PW, Chao ST, Sneed PK, et al. Diagnosis-specific prognostic factors, indexes, and treatment outcomes for patients with newly diagnosed brain metastases: a multi-institutional analysis of 4,259 patients. Int J Radiat Oncol Biol Phys 2010; 77: 655-661.

9 Sperduto PW, Yang TJ, Beal K, et al. Estimating survival in patients with lung cancer and brain metastases: an update of the Graded Prognostic Assessment for Lung Cancer Using Molecular Markers (Lung-molGPA). JAMA Oncol 2017; 3: 827-831.

10 Hendriks LEL, Henon C, Auclin E, et al. Outcome of patients with non-small cell lung cancer and brain metastases treated with checkpoint inhibitors. J Thorac Oncol 2019; 14: 1244-1254.

11 Zimmermann S, Dziadziuszko R, Peters S. Indications and limitations of chemotherapy and targeted agents in non-small cell lung cancer brain metastases. Cancer Treat Rev 2014; 40: 716-722.

12 Goldberg S, Gettinger S, Mahajan A, et al. Durability of brain metastasis response and overall survival in patients with non-small cell lung cancer (NSCLC) treated with pembrolizumab. J Clin Oncol 2018; 36: abstr 2009.

13 Goldberg SB, Gettinger SN, Mahajan A, et al. Pembrolizumab for patients with melanoma or non-small-cell lung cancer and untreated brain metastases: early analysis of a non-randomised, open-label, phase 2 trial. Lancet Oncol 2016; 17: 976-983.

14 Garassino M, Gadgeel S, Esteban E EF, et al. Abstract: Outcomes among patients (pts) with metastatic nonsquamous NSCLC with liver metastases or brain metastases treated with pembrolizumab (pembro) plus pemetrexed-platinum: results from the KEYNOTE-189 study. Cancer Res 2019; 79: Suppl., CT043.

15 Besse B, Le Moulec S, Mazieres J, et al. Bevacizumab in patients with nonsquamous non-small cell lung cancer and asymptomatic, untreated brain metastases (BRAIN): a nonrandomized, phase ii study. Clin Cancer Res 2015; 21: $1896-1903$.

16 Ilhan-Mutlu A, Osswald M, Liao $\mathrm{Y}$, et al. Bevacizumab prevents brain metastases formation in lung adenocarcinoma. Mol Cancer Ther 2016; 15: 702-710.

17 Socinski MA, Jotte RM, Cappuzzo F, et al. Atezolizumab for first-line treatment of metastatic nonsquamous NSCLC. N Engl J Med 2018; 378: 2288-2301.

18 Magnuson WJ, Lester-Coll NH, Wu AJ, et al. Management of brain metastases in tyrosine kinase inhibitor-naive epidermal growth factor receptor-mutant non-small-cell lung cancer: a retrospective multi-institutional analysis. J Clin Oncol 2017; 35: 1070-1077.

19 Hochstenbag MM, Twijnstra A, Hofman P, et al. MR-imaging of the brain of neurologic asymptomatic patients with large cell or adenocarcinoma of the lung. Does it influence prognosis and treatment? Lung cancer 2003; 42: 189-193.

20 Schoenmaekers J, Hofman P, Bootsma G, et al. Screening for brain metastases in patients with stage III non-small-cell lung cancer, magnetic resonance imaging or computed tomography? A prospective study. Eur J Cancer 2019; 115: 88-96. 
21 Gaspar LE, Chansky K, Albain KS, et al. Time from treatment to subsequent diagnosis of brain metastases in stage III non-small-cell lung cancer: a retrospective review by the Southwest Oncology Group. J Clin Oncol 2005; 23: $2955-2961$.

22 Remon J, Besse B. Brain metastases in oncogene-addicted non-small cell lung cancer patients: incidence and treatment. Front Oncol 2018; 8: 88.

23 Fallet V, Cadranel J, Doubre H, et al. Prospective screening for ALK: clinical features and outcome according to ALK status. Eur J Cancer 2014; 50: 1239-1246.

24 Pedrosa R, Mustafa DAM, Aerts J, et al. Potential molecular signatures predictive of lung cancer brain metastasis. Front Oncol 2018; 8: 159.

25 Mansfield AS, Aubry MC, Moser JC, et al. Temporal and spatial discordance of programmed cell death-ligand 1 expression and lymphocyte tumor infiltration between paired primary lesions and brain metastases in lung cancer. Ann Oncol 2016; 27: 1953-1958.

26 Mansfield AS, Ren H, Sutor S, et al. Contraction of T cell richness in lung cancer brain metastases. Sci Rep 2018; 8: 2171.

27 Ishihara D, Pop L, Takeshima T, et al. Rationale and evidence to combine radiation therapy and immunotherapy for cancer treatment. Cancer Immunol Immunother 2017; 66: 281-298.

28 Chen L, Douglass J, Kleinberg L, et al. Concurrent immune checkpoint inhibitors and stereotactic radiosurgery for brain metastases in non-small cell lung cancer, melanoma, and renal cell carcinoma. Int J Radiat Oncol Biol Phys 2018; 100: 916-925.

29 Hendriks LE, Schoenmaekers J, Zindler JD, et al. Safety of cranial radiotherapy concurrent with tyrosine kinase inhibitors in non-small cell lung cancer patients: a systematic review. Cancer Treat Rev 2015; 41: 634-645.

30 Hubbeling HG, Schapira EF, Horick NK, et al. Safety of combined PD-1 pathway inhibition and intracranial radiation therapy in non-small cell lung cancer. J Thorac Oncol 2018; 13: 550-558.

31 Martin AM, Cagney DN, Catalano PJ, et al. Immunotherapy and symptomatic radiation necrosis in patients with brain metastases treated with stereotactic radiation. JAMA Oncol 2018; 4: 1123-1124. 